\title{
Drug deposition in coronary arteries with overlapping drug-eluting stents
}

\author{
Farhad Rikhtegara, Elazer R. Edelman a,b, Ufuk Olgac ${ }^{\mathrm{c}}$, Dimos Poulikakos ${ }^{\mathrm{d}}$, \\ Vartan Kurtcuoglu ${ }^{\mathrm{c}, \mathrm{e}}$
}

Institute for Medical Engineering and Science, Massachusetts Institute of Technology, Cambridge, MA, USA

b Cardiovascular Division, Brigham and Women's Hospital, Harvard Medical School, Boston, MA, USA

c The Interface Group, Institute of Physiology, University of Zurich, Zurich, Switzerland

d Laboratory of Thermodynamics in Emerging Technologies, Department of Mechanical and Process Engineering, ETH Zurich, Zurich, Switzerland

e Zurich Center for Integrative Human Physiology and National Center of Competence 'Kidney.CH', University of Zurich, Zurich, Switzerland

Corresponding Author:

Farhad Rikhtegar

Harvard-MIT Biomedical Engineering Center

77 Massachusetts Avenue, Building E25-449,

Cambridge, MA 02139. USA

E-mail: $\quad$ farhadr@mit.edu

Phone: $\quad+1(617) 2588894$

Fax: $\quad+1(617) 2582514$

Word count: 5281

(C) 2016. This manuscript version is made available under the Elsevier user license 


\begin{abstract}
Drug-eluting stents are accepted as mainstream endovascular therapy, yet concerns for their safety may be under-appreciated. While failure from restenosis has dropped to below $5 \%$, the risk of stent thrombosis and associated mortality remain relatively high. Further optimization of drug release is required to minimize thrombosis risk while maintaining therapeutic dose.

The complex three-dimensional geometry of deployed stents together with the combination of diffusive and advective drug transport render an intuitive understanding of the situation exceedingly difficult. In situations such as this, computational modeling has proven essential, helping define the limits of efficacy, determine the mode and mechanism of drug release, and identify alternatives to avoid toxicity.

A particularly challenging conformation is encountered in coronary arteries with overlapping stents. To study hemodynamics and drug deposition in such vessels we combined highresolution, multi-scale ex vivo computed tomography with a flow and mass transfer computational model. This approach ensures high geometric fidelity and precise, simultaneous calculation of blood flow velocity, shear stress and drug distribution.

Our calculations show that drug uptake by the arterial tissue is dependent both on the patterns of flow disruption near the wall, as well as on the relative positioning of drug-eluting struts. Overlapping stent struts lead to localized peaks of drug concentration that may increase the risk of thrombosis. Such peaks could be avoided by anisotropic stent structure or asymmetric drug release designed to yield homogeneous drug distribution along the coronary artery and, at the least, suggest that these issues need to remain in the forefront of consideration in clinical practice.
\end{abstract}




\section{Introduction}

Drug eluting stents (DES) suppress neointimal hyperplasia and reduce angiographic restenosis seen in their bare metal stent (BMS) counterparts, but are not free of these disease processes [1-4]. DES angiographic and clinical restenosis increase with lesion complexity, and especially when multiple and overlapping stents are implanted [5]. The use of multiple stents and the intervention on long lesions virtually necessitate stent overlap, and is indeed present in more than one in three patients undergoing percutaneous coronary intervention (PCI) [6, 7]. Overlap is the one major category of interventions where new designs have not had as great an impact as seen in other use domains [8-10]. While some investigators have reported the safety and effectiveness of overlapping DES being comparable to those experienced with single long or multiple short stents [11-13], more recent publications have raised concerns about negative outcomes [14-18]. Regardless of stent type, stent overlap has been associated with increased instent restenosis (ISR) and lumen loss due to delayed healing and increased inflammation [6, 19, 20].

Stent overlap interferes with the two major goals of placement optimization - reduction in interference with luminal flow and minimization of mural injury. It is simply impossible to have complete apposition without exacerbating vascular injury, and/or minimal vascular injury without significant alteration in arterial hemodynamics. Stacking of stents creates regions of stagnation, separation and recirculation, the extent of which depends on stent geometry, asymmetric change of stent cell structure due to implantation and relative geometric configuration of overlapping struts [21-23]. These effects may result in wide variations in mural drug concentration with areas of depletion and excessive concentration $[22,24]$ exactly where precise control of drug delivery and retention is needed most - in the areas of injury. Overlapping struts or multiple struts in close proximity may increase the risk of local cytotoxicity, persistent inflammation [25], amplification of thrombogenic effects as a hypersensitivity reaction of the local vasculature to a specific polymer and/or drug [25, 26], 
stent thrombosis and in-stent restenosis $[6,21,27]$ as a consequence of increased arterial injury, poor endothelization and delayed healing [18, 25, 28].

The issues are thus profoundly complex, as we must now account for a balanced integration of drug delivery, vascular injury and flow disruption - considerations that defy intuition and are too costly to embrace in all its permutations in any experimental setting. This situation calls for a computational modeling approach with which the number of parameters as well as their scope can be reduced to experimentally tractable sizes.

Previous computational models have employed isolated struts devoid of complete designs, theoretical constructs and primarily two-dimensional systems [21, 24, 29, 30]. While they make a point, they fail to integrate the complete interactions between adjacent struts and the limitations imposed by real systems. More intricate designs of strut cells provide stents with more flexibility and ease of deployment and enhance the apposition even in case of stent overlap. However, in the presence of complex target lesions, malposition of struts is more pronounced especially in case of overlap, which results in greater disruption of near-wall flow [31]. Thus, simple consideration of two-dimensional primary systems, e.g. [24, 29, 30], will not allow for accurate modeling of the three-dimensional patterns of violated flow in arteries with overlapping stents.

Here we present a combined experimental and computational framework for the determination of hemodynamics and drug elution in stented arteries. Following a methodology we have developed and previously described in detail [32], we resolve the precise three dimensional geometry of stented arteries from microscale computed tomography data, ensuring high geometric fidelity both at the whole stent as well as at the individual strut scale. In conjunction with computational modeling, this allows prediction of drug distribution and deposition in anatomically accurate arteries under physiologic and pathophysiologic flow conditions. We show that taking into account the relative positioning of overlapping struts as 
well as arterial deformation warrants more realistic estimation of drug uptake by the tissue and highlights several hemodynamic and consequent pharmacodynamics outcomes which are the predecessors of reported clinical events.

\section{Methods}

\subsection{Ex vivo preparation of model: Stent implantation, vascular corrosion casting}

The left coronary artery of an ex vivo porcine heart was cannulated, and an interventional cardiologist implanted two absorbable magnesium alloy scaffolds of $10 \mathrm{~mm}$ length and $3 \mathrm{~mm}$ diameter (Biotronik AG, Bülach, Switzerland) under angiographic guidance. Care was taken to ensure reproducible $20 \%$ overlap of the stents. Note that in the computational model the scaffolds are treated as permanent drug eluting stents (see Section 2.3).

A radio-opaque casting material was prepared by mixing the low-shrinkage epoxy-based Biodur E20 resin (Biodur Products GmbH, Heidelberg, Germany) and iodine-saturated methyl ethyl ketone solvent. Using a pneumatic apparatus, the casting material was injected under physiological pressure of $90 \mathrm{mmHg}(120 \mathrm{mbar}$ ) to accurately capture the arterial tree. The heart was maintained at room temperature for 36 hours, and then macerated for $12 \mathrm{~h}$ at $55{ }^{\circ} \mathrm{C}$ in a $7.5 \% \mathrm{w} / \mathrm{v}$ solution of potassium hydroxide. The final product was rinsed with water several times to remove remaining adherent tissue.

A more detailed description of the ex vivo preparation can be found in $[31,32]$.

\subsection{Scanning and Image processing: $\mu \mathrm{CT}$ imaging of casts, segmentation and registration}

The overall geometry of the arterial tree was captured using micro-computed tomography ( $\mu$ CT 8o, Scanco Medical AG, Brüttisellen, Switzerland) with an isotropic voxel size of $74 \mu \mathrm{m}$ (energy $70 \mathrm{kVp}$, integration time $300 \mathrm{~ms}$, tube current $114 \mu \mathrm{A}$, and two times frame averaging). The stented arterial segment was dissected and re-scanned ( $\mu$ CT 40, Scanco) with an isotropic 
voxel size of $6 \mu \mathrm{m}$ (energy $70 \mathrm{kVp}$, integration time $300 \mathrm{~ms}$, tube current $114 \mu \mathrm{A}$, and two times frame averaging) to obtain higher resolution images. This strategy enabled us to resolve the individual stent struts as well as the overall arterial geometry while keeping scan time and associated costs at bay.

To partly suppress noise in the raw $\mu \mathrm{CT}$ volumes, a constrained $3 \mathrm{D}$ Gauss filter was applied ( $\sigma$ $=1.2, \mathrm{~S}=1.0$ ). Using a semi-automatic, intensity-based approach in Avizo 6.2 (Visualization Sciences Group SAS, Merignac, France), both $\mu \mathrm{CT}$ datasets of low and high resolution were independently segmented to obtain the lumen geometry. The resulting $3 \mathrm{D}$ geometry was exported to Geomagic Studio 12 (Geomagic, Inc., Morrisville, NC, USA), wherein the high resolution geometry of the stented segment and the lower resolution remainder of the arterial tree were registered and merged. Assuming 500 microns as a nominal value of a coronary artery wall thickness (smaller than human vascular thickness [33]), the vascular wall was generated by numerical expansion of the segmented lumen boundary. The obtained geometry was exported in STL format for subsequent computational grid generation.

\subsection{Computational set-up and governing equations}

ANSYS ICEM CFD (ANSYS, Inc., Canonsburg, PA, USA) was used to generate a computational grid of approximately 85 million tetrahedral elements in the merged geometry. A sequential CFD and mass transfer model was applied to conduct steady-state blood flow and mass transfer analysis for determining the blood velocity inside the artery and drug distribution in the lumen and the arterial wall. The following mass and momentum conservation equations were solved inside the lumen:

$$
\begin{gathered}
\nabla \cdot \overrightarrow{V_{l}}=0 \\
\rho\left(\overrightarrow{V_{l}} \cdot \nabla \overrightarrow{V_{l}}\right)=-\nabla P+\nabla \cdot\left(\mu \nabla \overrightarrow{V_{l}}\right)
\end{gathered}
$$


where $\vec{V}_{l}, \rho, P$ and $\mu$ are the blood flow velocity vector inside the lumen, density, pressure and dynamic viscosity, respectively. Blood was modeled as a non-Newtonian incompressible fluid with constant density of $1050 \mathrm{~kg} / \mathrm{m}^{3}$ and shear-dependent dynamic viscosity according to the Carreau model [34]. Blood was assumed to enter the coronary artery at a flow rate of $0.95 \mathrm{~mL} / \mathrm{s}$ at the ostium [35]. No slip boundary conditions were set at the stent wall, while on the vessel wall the transmural velocity of plasma was applied in the normal direction according to the precalculated luminal pressure distribution and resistance of the tissue excluding endothelial resistance as explained in [36, 37]. Murray's law was applied at the outlets, to where the outlet with the largest diameter was set to $70 \mathrm{mmHg}$ relative pressure, and the remaining outlets were assigned outflow rates according to their cross-sectional area [32, 38]. Drug transport inside the lumen was determined by the advection-diffusion equation

$$
\overrightarrow{V_{l}} \cdot \nabla C_{l}=D_{l} \cdot \nabla^{2} C_{l}
$$

where $C_{l}$ is the drug concentration within the fluid domain and $D_{l}$ denotes the diffusivity of the drug. Paclitaxel served as the model drug, with its diffusivity of $3.89 \times 10^{-11} \mathrm{~m}^{2} / \mathrm{s}$ in blood [39]. Zero drug concentration was assigned at the ostium. Open boundary conditions as well as flux conservation were assumed at the outlets and the mural interface, respectively. Constant unit concentration was set on the stent struts.

Similarly, the continuity and momentum conservation equations were solved for inside the arterial wall, which was modeled as a porous medium:

$$
\begin{gathered}
\nabla \cdot \overrightarrow{V_{w}}=0 \\
\rho\left(\overrightarrow{V_{w}} \cdot \nabla \overrightarrow{V_{w}}\right)=-\nabla P+\nabla \cdot\left(\mu \nabla \overrightarrow{V_{w}}\right)-\left(\frac{\mu}{K}\right) \overrightarrow{V_{w}}
\end{gathered}
$$

where $\overrightarrow{V_{w}}$ is infiltrated fluid velocity inside the arterial wall and momentum is updated according to Darcy's law with K, the permeability of wall, having the value of $1.43 \times 10^{-14} \mathrm{~cm}^{2}[39,40]$. No 
endothelial resistance was considered due to the assumption of complete endothelial denudation as a result of stenting [23]. Same normal transmural velocity (explained above) was applied as boundary condition at the luminal side of the arterial wall. Perivascular pressure was set to $17.5 \mathrm{mmHg}[36,37]$.

As in the lumen, drug transport inside the arterial wall is also governed by the advectiondiffusion equation,

$$
\overrightarrow{V_{w}} \cdot \nabla C_{w}=D_{w} \cdot \nabla^{2} C_{w}
$$

where $C_{w}$ is the concentration of the drug inside the artery wall and $D_{w}$ the corresponding drug diffusivity with the value of $3.65 \times 10^{-12} \mathrm{~m}^{2} / \mathrm{s}$ [39]. Open boundary conditions were prescribed at the perivascular border and the longitudinal ends of the considered arterial wall segment.

Calculations were carried out with the convergence criterion set to residual reduction to $10^{-8}$ of the initial value. 64 AMD Opteron 6174 processor cores were used. Grid independence studies were performed to ensure independence of the results from the used computational mesh. 


\section{Results}

In the following we present the calculated concentration of paclitaxel in the lumen and vascular wall of an ex vivo stented porcine left coronary artery. We show the effect of stent overlap in terms of flow disruption and thereby caused changes in drug distribution. To assess the effect of overlapping DES on vascular drug deposition we compare results of cases where only the proximal or distal stent (henceforth referred to as Proximal DES or Distal DES, respectively), or both elute drug (Overlap DES) (Figure 1).

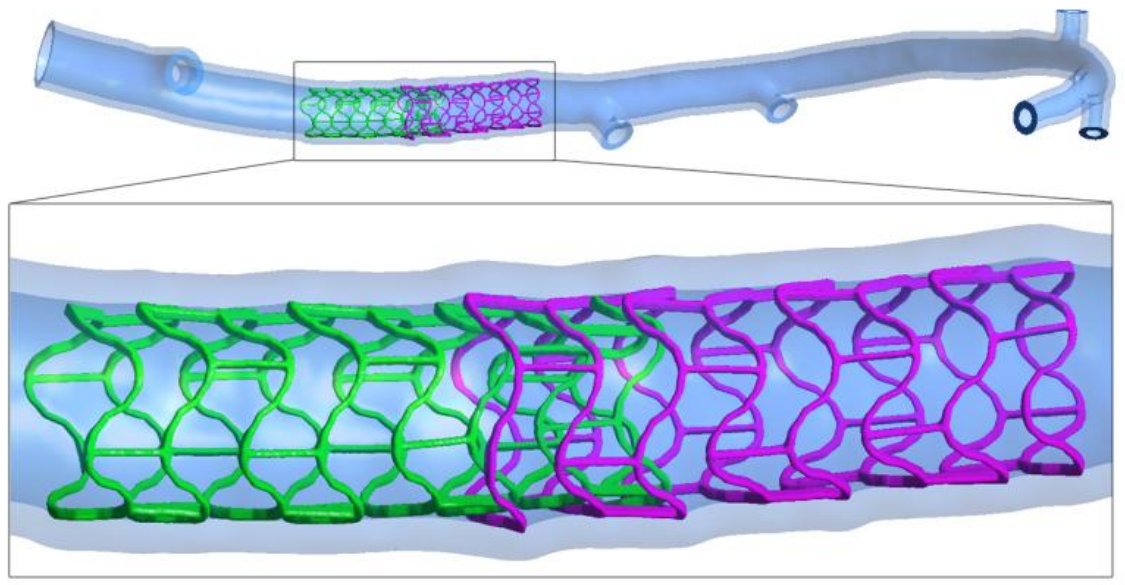

Figure 1. The overall geometry of stented porcine left coronary artery. The inset shows the stented region and overlap of the proximal, adluminally implanted, with the distal stent. The protrusion of the stent struts inside the vascular wall is evident.

Flow effects on mural drug deposition are increased in Overlap DES (Figure 2), the drug concentration is high in the vicinity of stent struts and at certain locations around the area of overlap. The recirculation zones and flow separation close to the struts induce an asymmetric drug deposition contour in the longitudinal flow direction in agreement with previous reports $[39,41]$. Stent overlap causes higher local drug peak concentrations which are expected to arise not only due to higher local drug load but also more direct contact of the drug eluting struts and tissue and, more important for us, disrupted blood flow. 


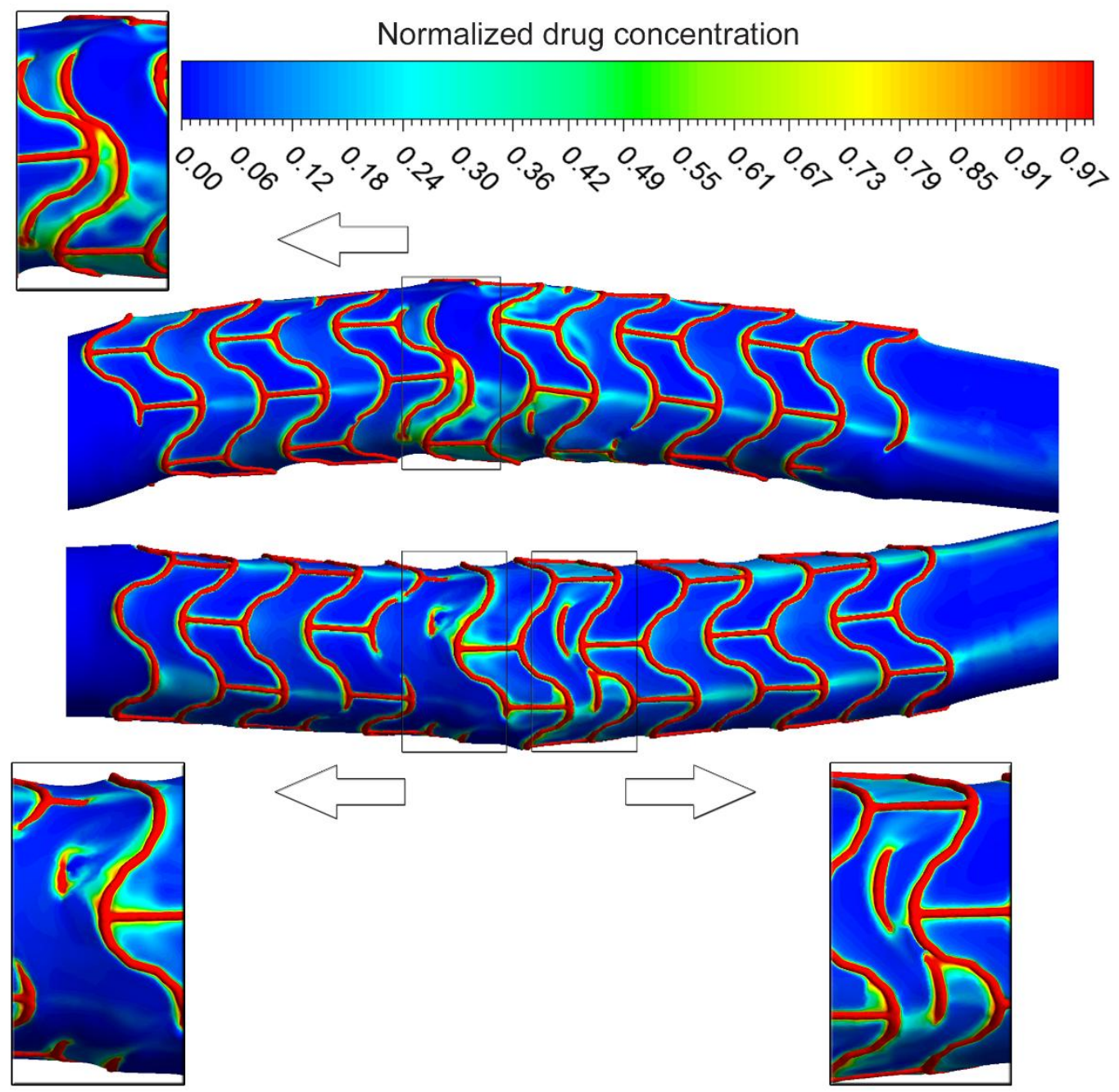

Figure 2. Mural drug deposition in an artery. Insets show magnified views of drug concentration pattern in different locations in the vicinity of stent overlap. Values are given relative to the drug concentration on the stent surface. Higher drug concentrations are evident in areas where the stent struts overlap. Asymmetric pattern of drug deposition and also signs of convectivelydelivered drug are observed

The relative strut configuration plays an important role in the drug deposition profile deep into the vessel wall (Figure 3) with higher concentration of drug at the site of overlap and in vicinity of inter-strut connectors. A large drug concentration gradient within the arterial wall is observed with concentrations peaking in the immediate vicinity of stent struts, and reducing with increasing distance from them. Furthermore, it appears that the luminal flow alleviates the 
extreme spatial heterogeneity of drug distribution that would be expected in the case of solely diffusive transport.

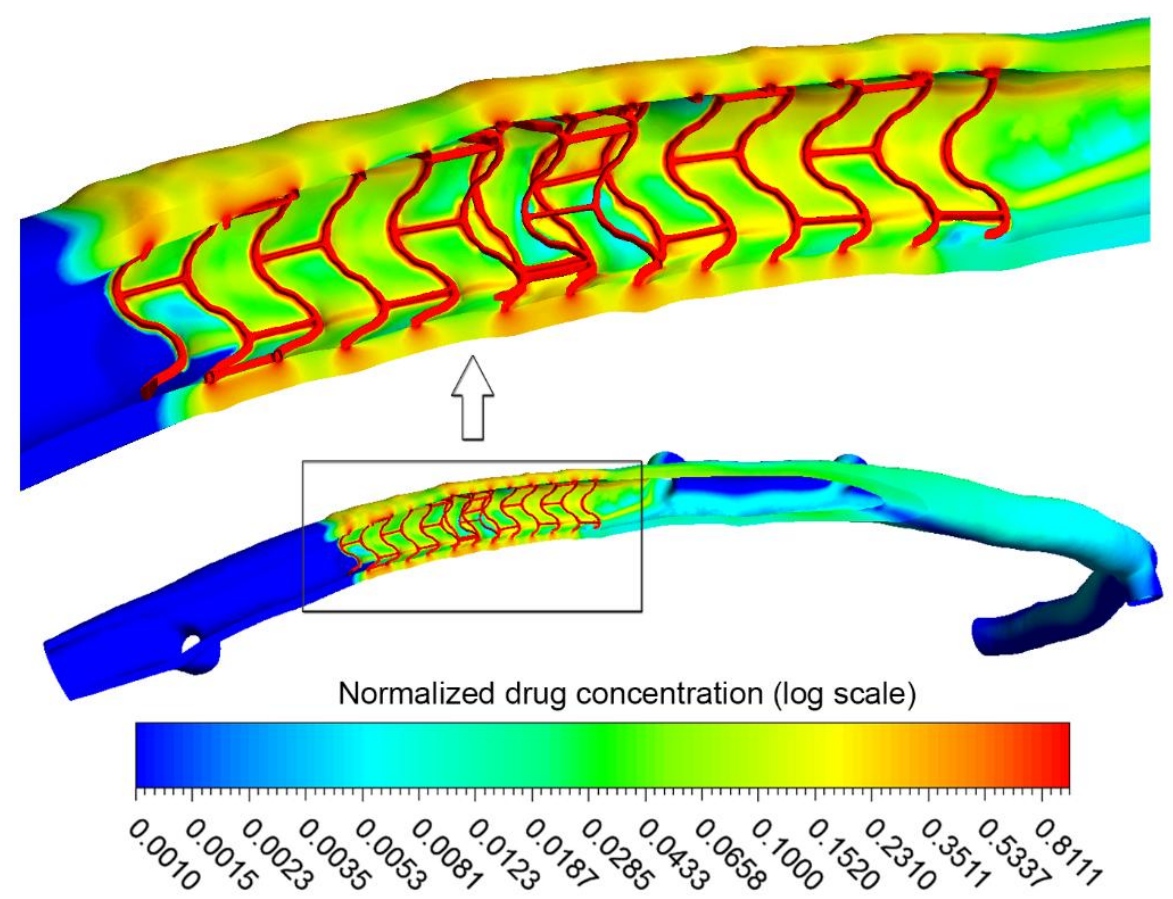

Figure 3. Concentration of deposited drug (shown in logarithmic scale) on the mural interface and in the arterial wall. Values are given relative to the drug concentration on the stent surface. Inset shows a high magnification view of the drug concentration pattern in the stented segment. High drug deposition is noticed in the overlap region, especially where struts are in close proximity, and distal to inter-strut connectors.

The flow-disrupting effect of overlapping struts extends drug well beyond the site of release (Figure 4A, B and C) - an effect more pronounced for cases with single drug-eluting stent. Drug concentration is notably high in locations with large flow recirculation zone caused by congruent overlapping stent struts. The area proximal to the overlap region is only marginally affected by flow disruption (Figure 4D), and in this region Proximal and Overlap DES cases differ in drug deposition only in the immediate vicinity of stent struts where small recirculation zones are generated. However, inside the overlap region (segments 2 and 3 in Figure 4D) large recirculation zones increase drug residence time and reduce flow-induced washout. Proximal to segment 3, blood can flow in between congruent struts since there is a comparably large 
distance between the inner- and outermost struts, increasing blood velocity, decreasing recirculation zone size and with it drug pooling. As a result, more drug is advected to the mural surface and into the arterial wall. Immediately distal to the overlap region (segment 4 ), the Overlap DES and Distal DES cases feature comparable drug concentration maps on the mural surface, with slightly higher concentration in the Overlap DES case due to drug elution from both stents. Interestingly, this leads to slightly higher concentration inside the tissue in the Distal DES case compared to when both stents are drug-eluting. Ultimately, the drug concentration just beneath congruent overlapping struts shows similar profile peaking right above the protruded strut for both Overlap and Distal DES cases. Moving farther in either proximal or distal direction, the flow-mediated effect of drug pooling and advective drug transport increases the drug concentration inside the tissue in the Overlap DES case. 
Normalized drug concentration

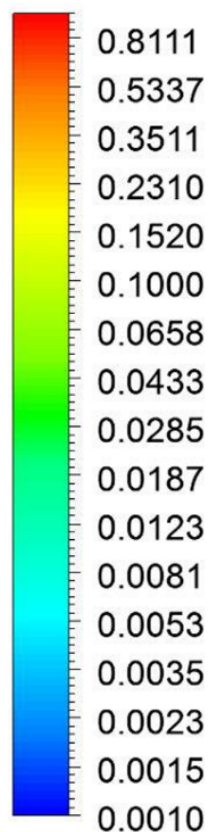

(log scale)

A

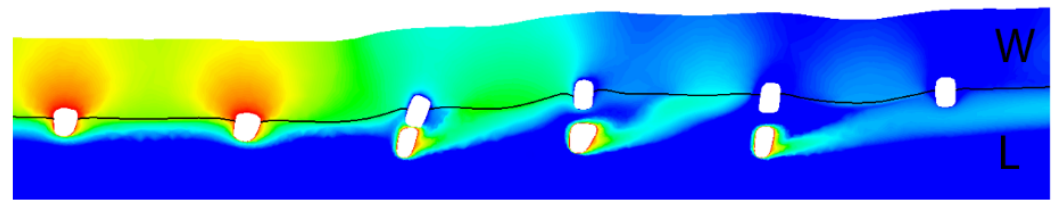

B
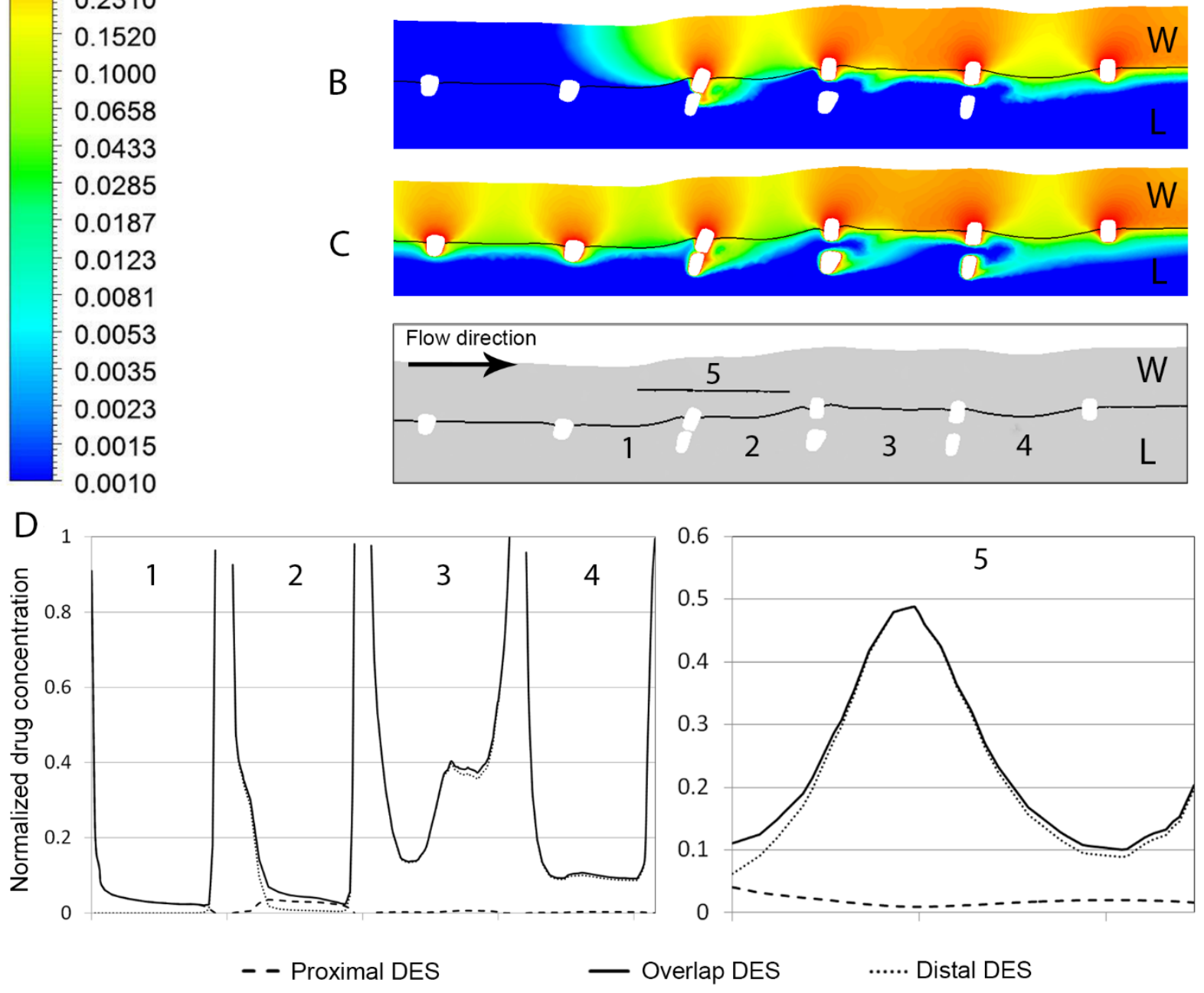

Figure 4. Drug concentration map in a section plane through two congruent struts in cases of drug elution by (A) the proximal (B) the distal and (C) both stents. Values are given relative to the drug concentration on the stent surface. W and L stand for the wall and lumen region, respectively. Drug pooling as a result of flow recirculation is evident at the site of overlap. (D) Plots of drug concentration along the lines (1) to (5) as defined in the section plane below panel (C). Lines (1) to (4) are located on the mural surface, whereas line (5) is midway inside the arterial wall right beneath two congruent struts.

To quantify the effect of overlap when the overlapping struts are both apposed to the artery, we compared the drug deposition on the mural interface and one half stent width into the tissue 
(Figure5). In the Overlap DES case (Figure 5C), the proximity of two drug-eluting struts not only increases the available drug load, but also generates a recirculation zone between the overlapping struts, which increases drug pooling and transfer to the tissue. The latter also increases drug concentration in cases where the proximal or distal strut does not elute drug (Distal/Proximal DES cases). Drug delivery is more effective when the drug-eluting stent is implanted abluminally (Figure 5A and B). On the mural wall, the drug concentration away from the stent struts is more than double the value found in the congruent case (Figure 4D and 6D). Moreover, the mural drug concentration is considerably higher when the drug-eluting stent is located distally. This shows that the drug concentration is mainly determined by flow-mediated effects. Overlapping drug-eluting struts in close proximity to each other act as one large obstacle, generating a larger recirculation zone proximal to the struts and thereby causing a slight shift in concentration pattern beneath the proximal strut (Figure 5D2). An interesting observation is also that while the Proximal DES case shows much lower mural concentration, the peak concentration in that case is higher compared to Distal DES as a result of flowmediated drug delivery caused by the larger drug pool proximal to the drug-eluting stent. While, from a qualitative point of view, these results are in line with observations of previous twodimensional simulations [24], the spatial variations seen here can only be obtained with threedimensional models. 


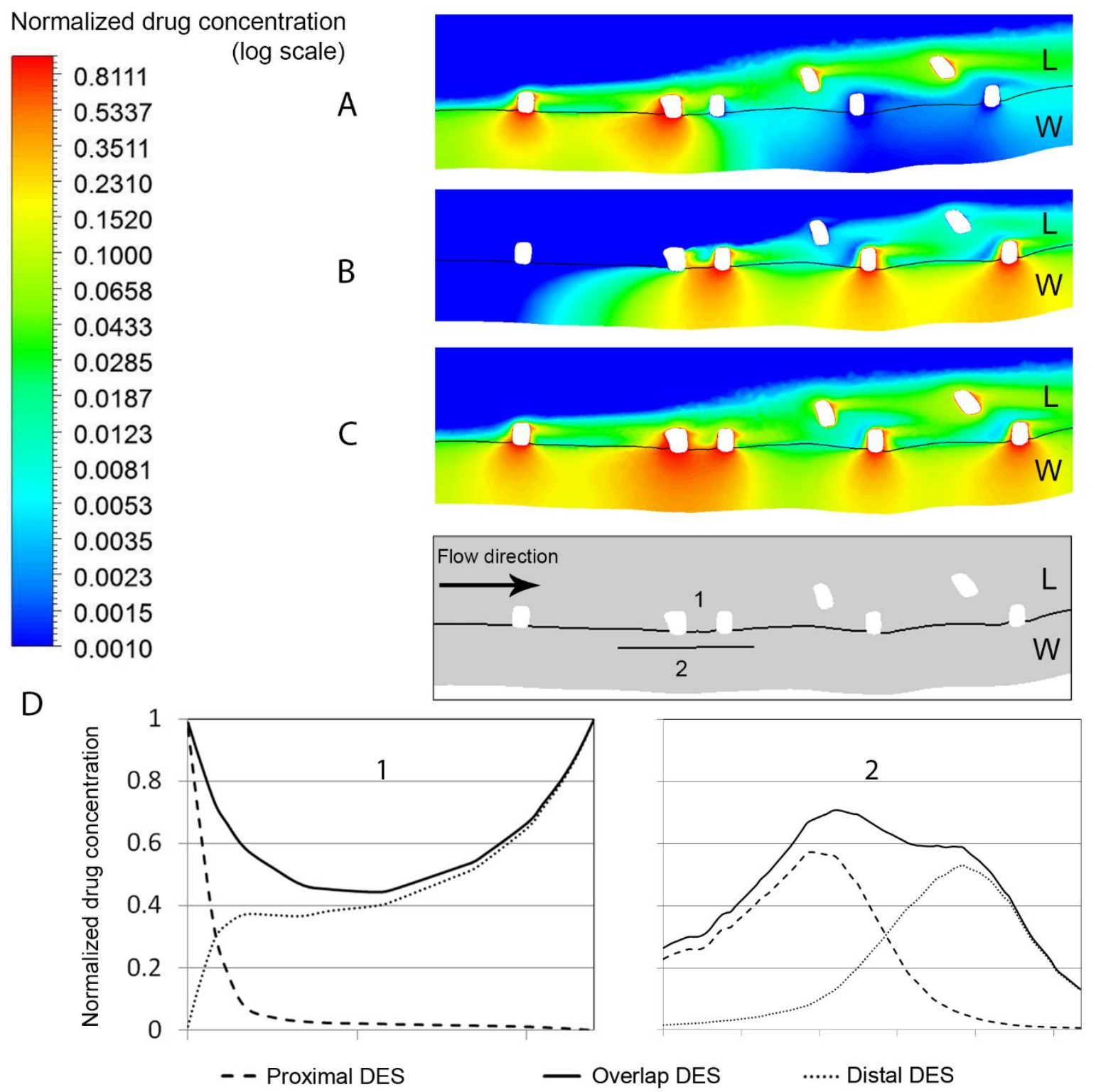

Figure 5. Drug concentration map in a plane of two abluminally apposed struts in different cases where (A) proximal (B) distal and (C) both stents are eluting drug. Values are given relative to the drug concentration on the stent surface. W and L stand for the wall and lumen region, respectively. Arterial drug uptake is considerably higher when both stents elute drug. (D) Plots of drug concentration on the mural surface and bellow two apposed overlapping struts, as defined in the inset. Line 1 and 2 are located on mural surface and inside arterial wall (halfwidth of strut width bellow) of apposed overlapping struts, respectively.

To further quantify the drug deposition map, Area-Weighted Average drug Concentration (AWAC) is calculated for the proximal, overlapping and distal segments of the stented region, 
plus for a single distal non-stented segment for the three cases of Proximal, Distal and Overlap DES (left panel of Figure6). The AWAC is always higher when both stents elute drug (Overlap DES). This dominance is minimized in the proximal segment, where the flow mediated difference and effect of overlap on drug load is negligible. In the overlap segment, the AWAC for Overlap DES is $420 \%$ and $34 \%$ higher than for Proximal and Distal DES, respectively. This shows that the flow-mediated effects increase the mural drug concentration considerably, but are more pronounced when the drug-eluting struts are abluminally implanted. It further shows that the second drug-eluting stent does not directly double the mural drug concentration, although the overall drug load is doubled. Finally, the highest mural concentration is found in the overlapping segment, where the availability of drug and flow disruption is maximized. For a non-stented region quite distal to the overlap segment, the AWAC is still higher for the case of Overlap DES, showing how the drug washed out by the flow alters drug deposition quite a distance from the strut overlap.
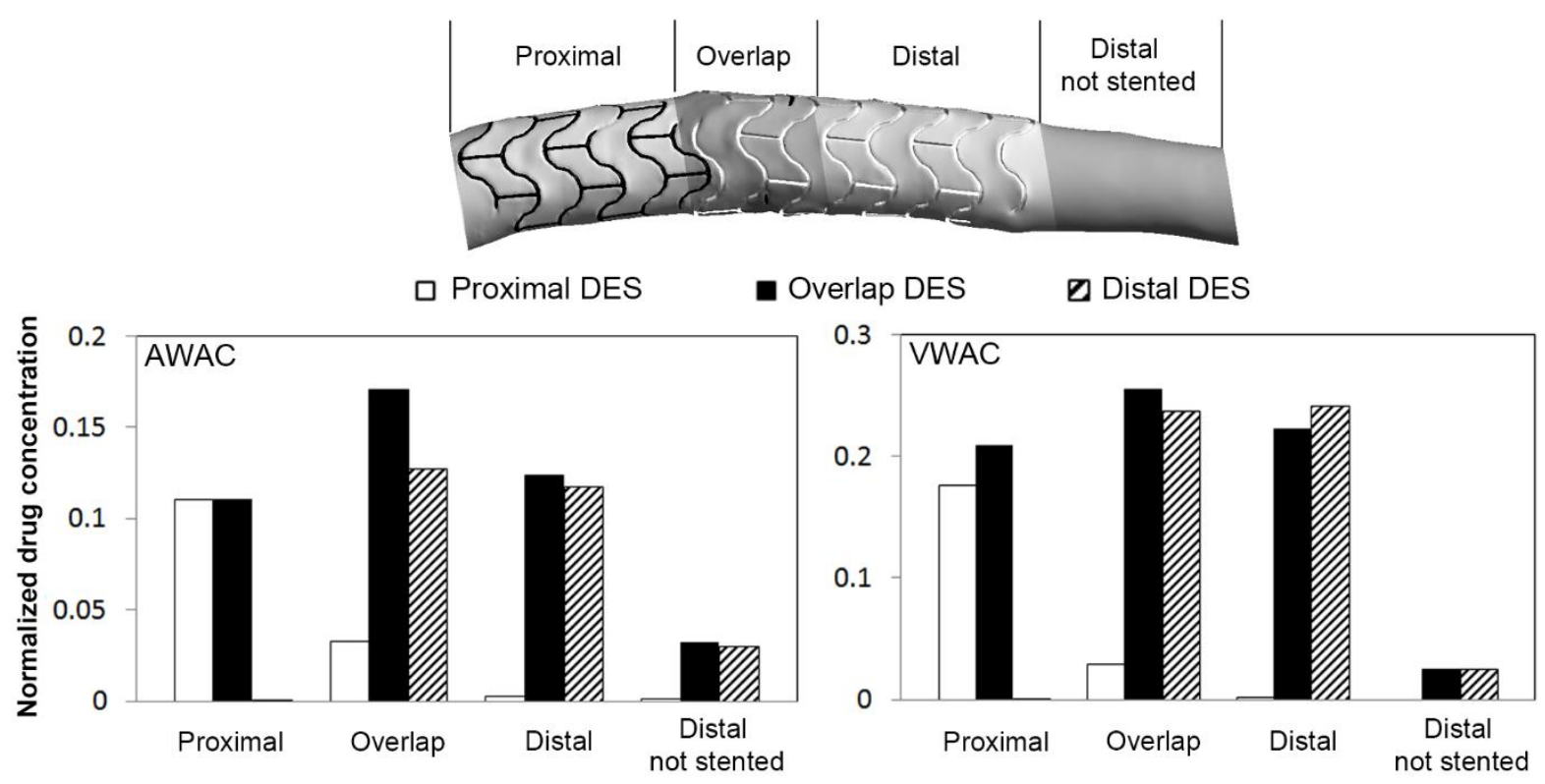

Figure 6. Quantification of the drug concentration on the mural surface and inside the wall of different segments of stented artery as defined in the schematic at the top. The values for the mural surface are given as the area weighted average of drug concentration (AWAC, left chart). For the arterial wall they are given as volume-weighted average of drug concentration (VWAC, right chart) within 
the arterial tissue. Values are given relative to the drug concentration on the stent surface. AWAC and VWAC are higher in the overlap segment than in the proximal and distal regions when both stents elute drug. This is due to higher load of drug available and flow-mediated drug deposition.

The total uptake of drug in the arterial wall, here expressed as Volume-Weighted Average drug Concentration (VWAC), is highest in the overlap region (Figure 6, right panel). The higher value of VWAC where the struts are abluminally apposed shows how crucial the direct contact of drug-eluting stent is to the accumulation of drug inside the arterial wall. The less pronounced difference between Overlap and Distal DES cases at the site of overlap highlights the dominance of direct contact over the flow-mediated drug delivery mechanism. Interestingly, drug uptake by the tissue is higher in the distal segment in the Distal DES compared to Overlap DES case. Observing tissue drug uptake in a location without stent contact (named 'Distal not stented'), we note a significant decrease in VAWC for comparably lower reduction of AWAC. This again highlights the importance of the drug delivery via direct contact compared to drug transfer by near-wall flow. 


\section{Discussions}

Overlapping stents have been compared to thick-strut devices, yet our results show that the issues with overlap extend well beyond the creation of recirculation zones. Our use of threedimensional modeling allows for control over geometry not possible in animal models, and enables the capture of features not observable in 2D models $[21,24,29,30]$. Arterial drug uptake is dependent on the patterns of flow disruption near the wall and the relative positioning of drug eluting struts. Overlapping struts localize drug concentration peaks. Three-dimensional modeling helps explain how a stent apposed to the artery wall can influence drug delivery from a second stent lying on top. The embedded struts of the apposed stent deliver substantial amount of drug to the wall due to direct contact. Net uptake by the tissue of drug released into the blood stream from the protruded struts of the second, overlapping stent depends on the drug concentration gradient across the luminal border. And this concentration gradient is directly related to the amount of drug delivered to the artery wall by the apposed stent.

The high spatial variation of drug deposition after DES implantation is caused by heterogeneous hemodynamics resulting from the interaction of local three-dimensional flow with anisotropic stent design, which ultimately leads to an asymmetric drug concentration map (Figure2) ) $[23,24,29,30]$. Interstrut spacing can now be visualized in $3 \mathrm{D}$ as an extension of overlap where struts adjacent to each other in the longitudinal domain can be understood to modulate the concertation gradient drive in a complex manner. Congruently overlapped struts, like individual thick struts, contribute to greater surface drug concentration drive into the tissue. This occurs in a manner where flow can play an even greater role as recirculation zones are expanded. However, the increase in drug deposition is lower than predicted by two-dimensional modeling [24], highlighting the limitation of the latter, simpler approach, and at the same time emphasizing the need for an accurate, three-dimensional account of hemodynamics and drug transport. When incongruent or staggered, the concentration gradients from individual struts can defeat each other and may not establish large recirculation zones (Figure 4 and Figure 5). 
Thus, we can now appreciate why drug deposition can be highly variable in areas distal or proximal to the stented segment - variable but not random: as our results indicate, the variability arises from the relative configuration of stent struts and inter-strut connectors due to the effect of convectively delivered drug. Hence, the overall drug uptake by the arterial tissue is not only determined by direct contact of drug eluting struts with the arterial wall, but also a result of flow-mediated transport.

In addition to increasing the direct contact between the arterial tissue and the drug-eluting struts, overlapping stents amplify the drug load in the vicinity due to alteration of blood flow. Strut embedment increases drug concentration in the wall and decreases the amount of drug advected to distal regions. On the other hand, protrusion of the stent struts disrupts the flow field and induces flow separation and recirculation zones that modulate the drug deposition in the vascular wall and on the mural surface. This phenomenon also alters drug delivery to regions between struts and minimizes the gap between local minimum and maximum drug concentration values. The resultant effect of increased drug deposition due to stent overlap greatly depends on the relative strut configuration, and is more pronounced, both on the mural surface and inside the tissue, when the struts of overlapping stents are positioned very close to each other. Indeed, since blood flow extends drug deposition beyond the area of stent-tissue contact, the complex interaction of stent struts with the arterial wall and the flow field needs to be taken into account. For instance in some cases, the peak concentration of delivered drug will not be just symmetrical beneath the implanted strut, as one might expect by intuition, but shifted towards the direction dictated by hemodynamic forces. Therefore, it may be prudent to put more emphasis on such flow effects in the stent design process and consider them during stent deployment. It is possible that some negative outcomes associated with DES may be linked to unexpected patterns of flow-induced drug deposition. 
In the case of stent overlap, malapposition and the effect of flow disruption will modulate the amount of drug uptake by the arterial tissue. While the amount of drug available at the luminal arterial surface increases with overlapping stents, it is not linearly dependent on the amount of drug on the stent struts, but co-determined by the local flow field (Figure 6). And again, there is large spatial variation in the flow field, which cannot be captured with a two-dimensional modeling approach.

The increase of drug deposited on the mural surface in case of overlap may lead to delayed vascular healing, which is a well-known issue in the treatment with DES [42]. This phenomenon, along with increased drug uptake distally and in inter-strut regions, highlights the complexity of the interaction between the flow-mediated distribution of released drug and drug uptake further downstream. If, in addition, one considers temporal changes caused by intimal hyperplasia, the complexity of the situation becomes even more pronounced. The important case of overlap, increasing the peak concentrations locally, may also complicate the total drug uptake due to the important role of local flow and inter-strut positioning. The overlap of stent struts and malapposition lead to considerable disruption of flow, extending drug delivery beyond the region of overlap. These near-wall flow-dependent features, although increasing the drug deposition on the mural wall as well as arterial drug uptake, will be more effective when the drug eluting struts are apposed to the arterial wall. Thus, in cases where the drug-eluting stent is not abluminally implanted, the overlap, and consequently flow disruption, will pool the drug in the vicinity of the mural surface and amass the drug on the lumen-tissue interface, but will not noticeably add to the volumetric concentration of drug inside the arterial wall, as the diffusiongoverned drug transport to the tissue is gradient dependent.

Considering the area-weighted average drug deposition (Figure 6), we can conclude that flowmediated transport induced by the positioning of stent struts in overlap segments increases the drug concentration on the mural surface and its deposition inside the arterial wall at the overlap 
segment. Highest drug delivery is achieved when both of overlapping stents elute drug, and this is more pronounced at the site of overlap. The overlap of stent struts, whether they are drugeluting or not, alters the drug deposition map more significantly at areas distal to the site of overlap than proximal. While strut overlap and flow-induced drug delivery affects predominantly drug deposition on the mural surface, drug uptake by arterial tissue is governed more by the direct contact of the stent struts. Although it is clear that the drug uptake by the tissue is indeed affected by the flow field, this effect is less important than the relative positioning and ab/adluminal installation of drug-eluting stents. Substantial drop of drug uptake by the arterial tissue at regions distal to the stented segment, despite comparably lesssignificant drop of luminal wall drug concentration (Figure 6), shows how crucial the role of stent direct contact is in arterial drug delivery. The non-zero drug uptake in these regions is, conversely, further proof of the direct impact of flow-induced heterogeneous drug delivery in areas well beyond the immediate reach of the stent struts.

Another factor of high clinical importance is the vascular injury induced by stent implantation. While increasing stent embedment would lead directly to augmented drug uptake, it would also lead to more vascular injury. In the complex scenario of stent overlap, the outer (first) stent is pushed further into the arterial wall, thereby causing more vascular injury, while the inner (second) stent causes more flow disturbance. Consequently, there will be localized peaks of drug concentration due to the outer stent's large area of contact with the tissue, while its contribution to advective drug transport will be decreased due to smaller area of contact with blood. In contrast, the inner stent will contribute more to drug transport by blood flow, also generating recirculation zones that pool the drug locally and lead to a non-uniform drug distribution map. This underlines the significance of the interventionist's decision between one long or multiple overlapping stents, and suggests that further considerations are needed for stent design and clinical follow up in cases with multiple stents. 


\subsection{Study limitations}

While our study has demonstrated very clearly that three-dimensional rather than twodimensional modeling is required to capture spatial variations of drug distribution, it is also evident that our framework does not account for all relevant physiologic and pathophysiologic mechanisms. An essential complement to this framework would be the consideration of biological variability and vascular responses [43]. This would include multi-scale modeling of drug pharmacokinetics and metabolism [44], as well as tissue partitioning and drug binding [45] in a heterogeneous tissue structure with more realistic diseased vessel topography. In those cases, the time-dependence of drug release [46] and the process of drug binding to tissue proteins will more accurately determine the ultimate drug uptake $[47,48]$. Assuming a more dynamic environment of biological host species, vascular response both to the intravascular implant and depleted drug in terms of intimal hyperplasia and thrombosis should also be included in future numerical models to better assess the efficacy of new generations of drugeluting implants [49] in more sophisticated clinical routines such as overlap [50, 51]. Developing such an intricate model entails further in vivo studies to acquire empirical factors of the biological response of the artery to develop a multi-scale, multi-modal tool. Nevertheless, simplified computational models, similar to the present approach, are still invaluable tools to reduce the number of contributing parameters and limit the search space for each playing factor to produce a viable experimental set-up and bring about an expedient platform to assess efficacy of endovascular implants and study their fundamental complexities. 


\section{Conclusions}

Combining two invaluable assets of our tool to first capture the geometry of individual struts as well as deformation of vasculature as a result of stenting and then computing point-wise maps of velocity, shear stress and drug concentration along the arterial tree, we were able to investigate complex geometrical configurations and diverse stenting scenarios. Arterial drug uptake and concentration patterns are highly dependent on both the local near-wall flow and the stent strut location and relative positioning. Realistic estimation of pharmacodynamics compels taking into account the three-dimensional characteristics of blood flow to predict the predecessors of reported clinical outcomes. Our $3 \mathrm{D}$ realistic computational model highlights the paramount role of both flow-mediated effects as well as geometrical positioning and design of drug eluting stents in the prediction of the drug delivery to the stented artery, which is more asymmetric and counter-intuitive in case of stent overlap. Apposition of drug-eluting struts and their geometrical characteristics dominated the drug distribution map, wherein relative positioning of struts from multiple stents and implantation procedure modulate the hemodynamics and consequently drug distribution.

We intend our framework to serve as a test bed for the development of new DES designs that will minimize adverse clinical events currently caused by suboptimal drug distribution. To this end, expansion of the computational model to include the more complex issues that surround use of these devices, e.g. time-dependent kinematics of drug release and retention of drug inside arterial tissue, is needed. Further aspects that warrant attention are the vasculature's response to the endovascular implant, heterogeneity of drug transport in different healthy and diseased vascular tissue layers, removal of drug by metabolism and correlation with clinical events from trials. 


\section{Acknowledgments}

The authors gratefully acknowledge the financial support provided by the Swiss National Science Foundation [grant number P2EZP3_155599], the National Institutes of Health (Ro1 GM 49039) and through the NCCR Kidney.CH. 
[1] W. Khan, S. Farah, A.J. Domb, Drug eluting stents: Developments and current status, Journal of Controlled Release, 161 (2012) 703-712.

[2] G.G. Stefanini, D.R.J. Holmes Drug-Eluting Coronary-Artery Stents, New England Journal of Medicine, 368 (2013) 254-265.

[3] T. Simard, B. Hibbert, F.D. Ramirez, M. Froeschl, Y.X. Chen, E.R. O'Brien, The Evolution of Coronary Stents: A Brief Review, Canadian Journal of Cardiology, 30 (2014) 35-45.

[4] G. Katz, B. Harchandani, B. Shah, Drug-eluting stents: the past, present, and future, Current atherosclerosis reports, 17 (2015) 485 .

[5] C.J. O'Sullivan, G.G. Stefanini, L. Raber, D. Heg, M. Taniwaki, B. Kalesan, T. Pilgrim, T. Zanchin, A. Moschovitis, L. Bullesfeld, A.A. Khattab, B. Meier, P. Wenaweser, P. Juni, S. Windecker, Impact of stent overlap on long-term clinical outcomes in patients treated with newer-generation drug-eluting stents, EuroIntervention : journal of EuroPCR in collaboration with the Working Group on Interventional Cardiology of the European Society of Cardiology, 9 (2014) 1076-1084.

[6] L. Räber, P. Juni, L. Loffel, S. Wandel, S. Cook, P. Wenaweser, M. Togni, R. Vogel, C. Seiler, F. Eberli, T. Luscher, B. Meier, S. Windecker, Impact of stent overlap on angiographic and long-term clinical outcome in patients undergoing drug-eluting stent implantation, Journal of the American College of Cardiology, 55 (2010) 1178-1188.

[7] E. Schampaert, J.W. Moses, J. Schofer, M. Schluter, A.H. Gershlick, E.A. Cohen, D.A. Palisaitis, G. Breithardt, D.J. Donohoe, H. Wang, J.J. Popma, R.E. Kuntz, M.B. Leon, Sirolimus-eluting stents at two years: a pooled analysis of SIRIUS, E-SIRIUS, and C-SIRIUS with emphasis on late revascularizations and stent thromboses, The American journal of cardiology, 98 (2006) 36-41.

[8] J.W. Moses, M.B. Leon, J.J. Popma, P.J. Fitzgerald, D.R. Holmes, C. O'Shaughnessy, R.P. Caputo, D.J. Kereiakes, D.O. Williams, P.S. Teirstein, J.L. Jaeger, R.E. Kuntz, Sirolimus-eluting stents versus standard stents in patients with stenosis in a native coronary artery, The New England journal of medicine, 349 (2003) 1315-1323. [9] M. Degertekin, P.W. Serruys, K. Tanabe, C.H. Lee, J.E. Sousa, A. Colombo, M.-C. Morice, J.M.R. Ligthart, P.J. de Feyter, Long-Term Follow-Up of Incomplete Stent Apposition in Patients Who Received Sirolimus-Eluting Stent for De Novo Coronary Lesions: An Intravascular Ultrasound Analysis, Circulation, 108 (2003) 2747-2750.

[10] E. Tsagalou, A. Chieffo, I. Iakovou, L. Ge, G.M. Sangiorgi, N. Corvaja, F. Airoldi, M. Montorfano, I. Michev, A. Colombo, Multiple overlapping drug-eluting stents to treat diffuse disease of the left anterior descending coronary artery, Journal of the American College of Cardiology, 45 (2005) 1570-1573.

[11] C.W. Lee, K.-H. Park, Y.-H. Kim, M.-K. Hong, J.-J. Kim, S.-W. Park, S.-J. Park, Clinical and angiographic outcomes after placement of multiple overlapping drug-eluting stents in diffuse coronary lesions, American Journal of Cardiology, 98 (2006) 918-922.

[12] J. Aoki, A.T.L. Ong, G.A.R. Granillo, E.P. McFadden, C.A.G. van Mieghem, M. Valgimigli, K. Tsuchida, G. Sianos, E. Regar, P.P.T. de Jaegere, W.J. van der Giessen, P.J. de Feyter, R.T. van Domburg, P.W. Serruys, "Full metal jacket" (stented length $>=64 \mathrm{~mm}$ ) using drug-eluting stents for de novo coronary artery lesions, American Heart Journal, 150 (2005) 994-999.

[13] W.W. Chu, P.K. Kuchulakanti, R. Torguson, B. Wang, L.C. Clavijo, W.O. Suddath, A.D. Pichard, L.F. Satler, K.M. Kent, R. Waksman, Comparison of Clinical Outcomes of Overlapping Sirolimus- Versus Paclitaxel-Eluting Stents in Patients Undergoing Percutaneous Coronary Intervention, The American journal of cardiology, 98 (2006) 1563-1566.

[14] P. Vermeersch, P. Agostoni, S. Verheye, P. Van den Heuvel, C. Convens, F. Van den Branden, G. Van Langenhove, R. Delayed, Increased late mortality after sirolimus-eluting stents versus bare-metal stents in diseased saphenous vein grafts, Journal of the American College of Cardiology, 50 (2007) 261-267.

[15] R. Moreno, C. Fernandez, R. Hernandez, F. Alfonso, D.J. Angiolillo, M. Sabate, J. Escaned, C. Banuelos, A. Fernandez-Ortiz, C. Macaya, Drug-eluting stent thrombosis - Results from a pooled analysis including 10 randomized studies, Journal of the American College of Cardiology, 45 (2005) 954-959.

[16] G. Nakazawa, S.K. Yazdani, A.V. Finn, M. Vorpahl, F.D. Kolodgie, R. Virmani, Pathological Findings at Bifurcation LesionsThe Impact of Flow Distribution on Atherosclerosis and Arterial Healing After Stent Implantation, Journal of the American College of Cardiology, 55 (2010) 1679-1687.

[17] D. Matsumoto, J. Shite, T. Shinke, H. Otake, Y. Tanino, D. Ogasawara, T. Sawada, O.L. Paredes, K.-i. Hirata, M. Yokoyama, Neointimal coverage of sirolimus-eluting stents at 6-month follow-up: evaluated by optical coherence tomography, European Heart Journal, 28 (2007) 961-967.

[18] T. Shinke, J. Li, J.P. Chen, L. Pendyala, T. Goodchild, R. Jabara, S. Geva, T. Ueno, N. Chronos, K. Robinson, D. Hou, High Incidence of Intramural Thrombus After Overlapping Paclitaxel-Eluting Stent Implantation Angioscopic and Histopathologic Analysis in Porcine Coronary Arteries, Circulation-Cardiovascular Interventions, 1 (2008) 28-35.

[19] K. Wang, X.R. Zhou, E. Verbeken, Q.B. Ping, Y.M. Huang, J.H. Huang, F. Van de Werf, I. De Scheerder, Overlapping coronary stents result in an increased neointimal hyperplasia: Insight from a porcine coronary stent model, J Interv Cardiol, 13 (2000) 173-177.

[20] D.J. Kereiakes, H. Wang, J.J. Popma, R.E. Kuntz, D.J. Donohoe, J. Schofer, E. Schampaert, B. Meier, M.B. Leon, J.W. Moses, Periprocedural and Late Consequences of Overlapping Cypher Sirolimus-Eluting Stents: Pooled Analysis of Five Clinical Trials, Journal of the American College of Cardiology, 48 (2006) 21-31.

[21] K. Kolandaivelu, R. Swaminathan, W.J. Gibson, V.B. Kolachalama, K.-L. Nguyen-Ehrenreich, V.L. Giddings, L. Coleman, G.K. Wong, E.R. Edelman, Stent Thrombogenicity Early in High-Risk Interventional Settings Is Driven by Stent Design and Deployment and Protected by Polymer-Drug Coatings, Circulation, 123 (2011) 14001409. 
[22] A.M. Salam, J. Al Suwaidi, D.R. Holmes, Drug-eluting coronary stents, Current Problems in Cardiology, 31 (2006) 8-119.

[23] C.W. Hwang, D. Wu, E.R. Edelman, Physiological transport forces govern drug distribution for stent-based delivery, Circulation, 104 (2001) 600-605.

[24] B. Balakrishnan, A.R. Tzafriri, P. Seifert, A. Groothuis, C. Rogers, E.R. Edelman, Strut position, blood flow, and drug deposition - Implications for single and overlapping drug-eluting stents, Circulation, 111 (2005) 29582965.

[25] A.V. Finn, F.D. Kolodgie, J. Harnek, L.J. Guerrero, E. Acampado, K. Tefera, K. Skorija, D.K. Weber, H.K. Gold, R. Virmani, Differential Response of Delayed Healing and Persistent Inflammation at Sites of Overlapping Sirolimus- or Paclitaxel-Eluting Stents, Circulation, 112 (2005) 270-278.

[26] W.J. van der Giessen, A.M. Lincoff, R.S. Schwartz, H.M.M. van Beusekom, P.W. Serruys, D.R. Holmes, S.G. Ellis, E.J. Topol, Marked Inflammatory Sequelae to Implantation of Biodegradable and Nonbiodegradable Polymers in Porcine Coronary Arteries, Circulation, 94 (1996) 1690-1697.

[27] M. Joner, A.V. Finn, A. Farb, E.K. Mont, F.D. Kolodgie, E. Ladich, R. Kutys, K. Skorija, H.K. Gold, R. Virmani, Pathology of drug-eluting stents in humans - Delayed healing and late thrombotic risk, Journal of the American College of Cardiology, 48 (2006) 193-202.

[28] S.Y. Lim, M.H. Jeong, S.J. Hong, D.S. Lim, J.Y. Moon, Y.J. Hong, J.H. Kim, Y. Ahn, J.C. Kang, Inflammation and delayed endothelization with overlapping drug-eluting stents in a porcine model of in-stent restenosis, Circulation Journal, 72 (2008) 463-468.

[29] B. Balakrishnan, J. Dooley, G. Kopia, E.R. Edelman, Thrombus causes fluctuations in arterial drug delivery from intravascular stents, Journal of Controlled Release, 131 (2008) 173-180.

[30] B. Balakrishnan, J.F. Dooley, G. Kopia, E.R. Edelman, Intravascular drug release kinetics dictate arterial drug deposition, retention, and distribution, Journal of Controlled Release, 123 (2007) 100-108.

[31] F. Rikhtegar, C. Wyss, K.S. Stok, D. Poulikakos, R. Müller, V. Kurtcuoglu, Hemodynamics in coronary arteries with overlapping stents, Journal of Biomechanics, 47 (2014) 505-511.

[30] F. Rikhtegar, C. Wyss, K.S. Stok, H. Ge, R.J. Choo, A. Ferrari, D. Poulikakos, R. Müller, V. Kurtcuoglu, Compound ex vivo and in silico method for hemodynamic analysis of stented arteries, PLoS One, 8 (2013) e58147.

[33] C.J. van Andel, P.V. Pistecky, C. Borst, Mechanical properties of porcine and human arteries: Implications for coronary anastomotic connectors, Annals of Thoracic Surgery, 76 (2003) 58-64.

[34] S. Chien, S. Usami, H.M. Taylor, J.L. Lundberg, Gregerse.Mi, Effects of hematocrit and plasma proteins on human blood rheology at low shear rates, Journal of Applied Physiology, 21 (1966) 81-87.

[35] R.M. Berne, Levy, M.N., Cardiovascular Physiology 5th ed, Mosby, St. Louis, 1986.

[36] U. Olgac, V. Kurtcuoglu, D. Poulikakos, Computational modeling of coupled blood-wall mass transport of LDL: effects of local wall shear stress, American journal of physiology. Heart and circulatory physiology, 294 (2008) H909-919.

[37] U. Olgac, D. Poulikakos, S.C. Saur, H. Alkadhi, V. Kurtcuoglu, Patient-specific three-dimensional simulation of LDL accumulation in a human left coronary artery in its healthy and atherosclerotic states, American journal of physiology. Heart and circulatory physiology, 296 (2009) H1969-1982.

[38] C.D. Murray, The Physiological Principle of Minimum Work. I. The Vascular System and the Cost of Blood Volume, Proceedings of the National Academy of Sciences of the United States of America, 12 (1926) 207-214.

[39] V.B. Kolachalama, E.G. Levine, E.R. Edelman, Luminal Flow Amplifies Stent-Based Drug Deposition in Arterial Bifurcations, PloS one, 4 (2009).

[40] S. Tada, J. Tarbell, Fenestral Pore Size in the Internal Elastic Lamina Affects Transmural Flow Distribution in the Artery Wall, Annals of Biomedical Engineering, 29 (2001) 456-466.

[41] V.B. Kolachalama, A.R. Tzafriri, D.Y. Arifin, E.R. Edelman, Luminal flow patterns dictate arterial drug deposition in stent-based delivery, Journal of Controlled Release, 133 (2009) 24-30.

[42] S. Windecker, B. Meier, Late Coronary Stent Thrombosis, Circulation, 116 (2007) 1952-1965.

[43] M.Y. Maslov, E.R. Edelman, A.E. Wei, M.J. Pezone, M.A. Lovich, High concentrations of drug in target tissues following local controlled release are utilized for both drug distribution and biologic effect: an example with epicardial inotropic drug delivery, J Control Release, 171 (2013) 201-207.

[44] A.D. Levin, M. Jonas, C.-W. Hwang, E.R. Edelman, Local and systemic drug competition in drug-eluting stent tissue deposition properties, Journal of Controlled Release, 109 (2005) 236-243.

[45] V.B. Kolachalama, S.D. Pacetti, J.W. Franses, J.J. Stankus, H.Q. Zhao, T. Shazly, A. Nikanorov, L.B. Schwartz, A.R. Tzafriri, E.R. Edelman, Mechanisms of Tissue Uptake and Retention in Zotarolimus-Coated Balloon Therapy, Circulation, 127 (2013) 2047-2055.

[46] A. Kraitzer, L. Ofek, R. Schreiber, M. Zilberman, Long-term in vitro study of paclitaxel-eluting bioresorbable core/shell fiber structures, Journal of Controlled Release, 126 (2008) 139-148.

[47] A.R. Tzafriri, A. Groothuis, G.S. Price, E.R. Edelman, Stent elution rate determines drug deposition and receptor-mediated effects, J Control Release, 161 (2012) 918-926.

[48] C.C. O'Brien, V.B. Kolachalama, T.J. Barber, A. Simmons, E.R. Edelman, Impact of flow pulsatility on arterial drug distribution in stent-based therapy, J Control Release, 168 (2013) 115-124.

[49] Y. Huang, H.C.A. Ng, X.W. Ng, V. Subbu, Drug-eluting biostable and erodible stents, Journal of Controlled Release, 193 (2014) 188-201.

[50] A.R. Tzafriri, N. Vukmirovic, V.B. Kolachalama, I. Astafieva, E.R. Edelman, Lesion Complexity Determines Arterial Drug Distribution After Local Drug Delivery, Journal of controlled release : official journal of the Controlled Release Society, 142 (2010) 332-338.

[51] W.C. Carlyle, J.B. McClain, A.R. Tzafriri, L. Bailey, B.G. Zani, P.M. Markham, J.R. Stanley, E.R. Edelman, Enhanced drug delivery capabilities from stents coated with absorbable polymer and crystalline drug, J Control Release, 162 (2012) 561-567. 


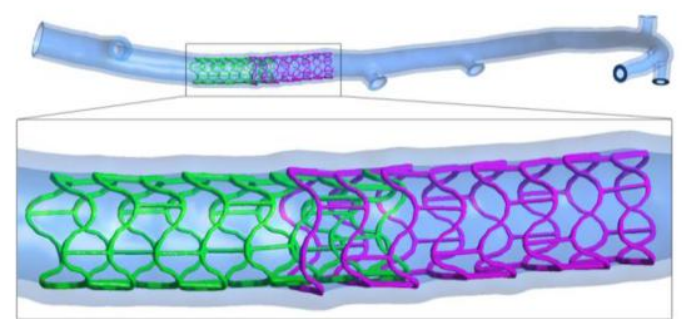

Realistic 3D reconstruction of artery and overlapping stents

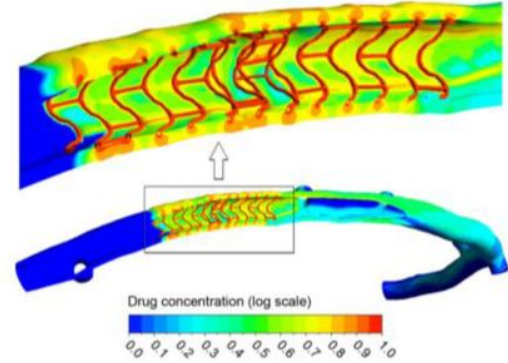

3D map of drug distribution on mural wall and inside arterial tissue 